\title{
Probabilistic Teleportation of an Arbitrary Two-Qubit State via Two Partially Entangled States
}

\author{
Xu Yi-Qiong ${ }^{1}$, Feng Zhi-Yong ${ }^{1}$, Wang $\mathrm{Bo}^{2}$, Shi $\mathrm{Lei}^{3}$, Liang Rong-Di ${ }^{3}$, Wei \\ Jia-Hua ${ }^{3}$
}
1 School of Information and CommunicationEngineering, Beijing University of Posts and Telecommunication, Beijing, 100876

2 Beijing JunChangShengAn Information Technology co., Itd, Beijing, 100095

3 Information and Navigation College,Air Force Engineering University,Shanxi Xi'An, 710073

*Email:weijiahua@126.com

\begin{abstract}
Key words: non-maximally entangled state, probabilistic teleportation, arbitrary two-qubit state Abstract: We propose a novel scheme to probabilistically transmit an arbitraryunknown two-qubit quantum state with the help of two partially entangled states. In this scheme, theteleportation with one sender and two receives can be realized when theinformation of non-maximally entangled states is only available for thesender. The original state would been teleported on the particles of the two senders. Furthermore, the concrete implementation processes of this proposal are presented, meanwhile the classical communication cost and thesuccessful probability of our scheme are calculated.
\end{abstract}

\section{Introduction}

Quantum transmission is presented by C.H. Bennett et al in 1993 [1]. Based on the quantum entanglement and measurement properties, the quantum mechanics, the specific nature of the collapse of contact transmission can be used in the case of not to send any quantum information, one can use the local operation and classical communication to realize the transmission of an unknown quantum state when the senders and receivers do not have any information about the quantum state. With the development of quantum teleportation, people gradually realize that quantum teleportation plays an important role in the field of quantum information, and lots of fruitful theoretical and experimental research results have been received[2-6]. W.L. Li et al presented how to probabilistically teleport an arbitrary unknown two-qubit quantum state via partially entangled states [7]. H.Y. Dai presented Probabilistic Teleportation based on two W states [8] or the combination of a GHZ state and a W state [9], in which a quantum state can be transmitted from the sender to one of two receivers. In 2004, the U.S. National Institute of Standards and Technology D. Winland et al explored how to implement quantum teleportation based on atomic entangled state. In 2007, the group of the European Space Agency and the university of Vienna performed successfully the teleportation of a single photon. Although lots of progresses of quantum teleportation in the aspect of theory research and physical experiment has been made, but there are still many open issues worthy of further research.

In the early stage for the teleportation of two particles, the receivers not only are required to introduce some auxiliary particles to carry on the corresponding unitary operation, but also need to master the relevant information of quantum entanglement. Obviously, in the case of that entanglement information are only useful for the sender, the former methods could not be achieved. This paper puts forward a new protocol of quantum teleportation for two particles to overcome the shortcomings, namely, the teleportation can be realized when only the sender has entangled information. The quantum entanglement channel is composed of two non-maximally entangled states, the communication nodes contains a sender and two receivers. After the successful completion of the teleportation, two receivers have the whole information of the transmitted state. At the same time, this paper gives the implementation steps of this protocol, and calculate the successful probability and the classical information cost for this novel scheme. The above work has a promoting effect to expand the research of quantum teleportation. 


\section{The teleportation when the sender has quantum channel}

This section would present how to realize the teleportation when only the sender has the information of entangled state. Suppose that the transmitted two-particle state can be expressed as:

$$
|\varphi\rangle_{12}=\alpha|00\rangle_{12}+\beta|01\rangle_{12}+\gamma|10\rangle_{12}+\delta|11\rangle_{12}
$$

here the complex numbers $\alpha, \beta, \gamma$ and $\delta$ satisfy the normalization formula $|\alpha|^{2}+|\beta|^{2}+|\gamma|^{2}+|\varepsilon|^{2}=1$. Quantum channel is composed of two non-maximally two-particle entangled states:

$$
\begin{aligned}
& |\varphi\rangle_{34}=a|00\rangle_{34}+b|11\rangle_{34} \\
& |\varphi\rangle_{56}=c|00\rangle_{56}+d|11\rangle_{56}
\end{aligned}
$$

where non-zero real numbers $a, c$ and the complex ones $b, d$ respectively satisfy $|\alpha|^{2}+|b|^{2}=1$ and $|c|^{2}+|d|^{2}=1$. Moreover, the inequalities $|a| \geq|b| \geq 0$ and $|c| \geq|d|>0$ are satisfied. Particle 3 and 5 belong to the sender Alice, the receivers Bob and Charlie have particle 4 and 6 , respectively. The implementation process of this proposal can be divided into four steps:

Step 1 Since quantum channel is composed of two non-maximally entangled states, some auxiliary particles need to be introduced in the implementation process. In this article, the sender Alice introduces two particle $m$ and $n$ with the initial state $|00\rangle_{m n}$. Hence, the overall state of particle system can be presented as:

$$
\left|\varphi^{0}\right\rangle_{123456 m i n}=|\varphi\rangle_{12} \otimes|\varphi\rangle_{34} \otimes|\varphi\rangle_{56} \otimes|00\rangle_{m m}
$$

Step 2 For particles $(1, m)$ and $(2, n)$, the sender Alice performs $4 \times 4$ unitary operations $U(a, b)$ and $U(c, d)$ :

$$
U(x, y)=\left(\begin{array}{cccc}
y / x & -\sqrt{1-|y / x|^{2}} & 0 & 0 \\
\sqrt{1-|y / x|^{2}} & y / x & 0 & 0 \\
0 & 0 & 1 & 0 \\
0 & 0 & 0 & 1
\end{array}\right) x=a, c
$$

After that , the state of particle system would be rewritten as:

$$
\begin{aligned}
& \left|\varphi^{0}\right\rangle_{123456 m n}=\left[U(a, b)\left|\varphi^{0}\right\rangle_{1 m}\right] \otimes\left[U(c, d)\left|\varphi^{0}\right\rangle_{2 n}\right] \otimes\left|\varphi^{0}\right\rangle_{3456} \\
& =p_{1}|00\rangle_{m e} \otimes\left|\Phi^{+}\right\rangle_{13} \otimes\left|\Phi^{+}\right\rangle_{25} \otimes(\alpha|00\rangle+\beta|01\rangle+\gamma|10\rangle+\delta|11\rangle)_{46} \\
& +p_{1}|00\rangle_{m n} \otimes\left|\Phi^{+}\right\rangle_{13} \otimes\left|\Phi^{-}\right\rangle_{25} \otimes(\alpha|00\rangle-\beta|01\rangle+\gamma|10\rangle-\delta|11\rangle)_{46} \\
& +p_{1}|00\rangle_{m n} \otimes\left|\Phi^{+}\right\rangle_{13} \otimes\left|\Psi^{+}\right\rangle_{25} \otimes(\alpha|01\rangle+\beta|00\rangle+\gamma|11\rangle+\tilde{\delta}|10\rangle)_{46} \\
& +p_{1}|00\rangle_{m n} \otimes\left|\Phi^{+}\right\rangle_{13} \otimes\left|\Psi^{-}\right\rangle_{25} \otimes(\alpha|01\rangle-\beta|00\rangle+\gamma|11\rangle-\delta|10\rangle)_{46} \\
& +p_{1}|00\rangle_{m n} \otimes\left|\Phi^{-}\right\rangle_{13} \otimes\left|\Phi^{+}\right\rangle_{25} \otimes(\alpha|00\rangle+\beta|01\rangle-\gamma|10\rangle-\delta \mid 11)_{46} \\
& +p_{1}|00\rangle_{m n} \otimes\left|\Phi^{-}\right\rangle_{13} \otimes\left|\Phi^{-}\right\rangle_{25} \otimes(\alpha|00\rangle-\beta|01\rangle-\gamma|10\rangle+\delta \mid 11)_{46} \\
& \left.\left.+p_{1}|00\rangle_{m n} \otimes\left|\Phi^{-}\right\rangle_{13} \otimes\left|\Psi^{+}\right\rangle_{25} \otimes(\alpha \mid 01)+\beta|00\rangle-\gamma|11\rangle-\delta \mid 10\right)\right\rangle_{46} \\
& \left.+p_{1}|00\rangle_{m n} \otimes\left|\Phi^{-}\right\rangle_{13} \otimes\left|\Psi^{-}\right\rangle_{25} \otimes(\alpha|01\rangle-\beta|00\rangle-\gamma|11\rangle+\delta \mid 10)\right\rangle_{46} \\
& \left.+p_{1}|00\rangle_{m n} \otimes\left|\Psi^{+}\right\rangle_{13} \otimes\left|\Phi^{+}\right\rangle_{25} \otimes(\alpha \mid 10)+\beta|11\rangle+\gamma|00\rangle+\delta|01\rangle\right\rangle_{46} \\
& \left.+p_{1}|00\rangle_{m n} \otimes\left|\Psi^{+}\right\rangle_{13} \otimes\left|\Phi^{-}\right\rangle_{25} \otimes(\alpha \mid 10)-\beta|11\rangle+\gamma|00\rangle-\delta|01\rangle\right\rangle_{46} \\
& +p_{1}|00\rangle_{m n} \otimes\left|\Psi^{+}\right\rangle_{13} \otimes\left|\Psi^{+}\right\rangle_{25} \otimes(\alpha|11\rangle+\beta|10\rangle+\gamma|01\rangle+\delta|00\rangle\rangle_{46} \\
& +p_{1}|00\rangle_{m n} \otimes\left|\Psi^{+}\right\rangle_{13} \otimes\left|\Psi^{-}\right\rangle_{25} \otimes(\alpha|11\rangle-\beta|10\rangle+\gamma|01\rangle-\delta|00\rangle\rangle_{46} \\
& +p_{1}|00\rangle_{m n} \otimes\left|\Psi^{-}\right\rangle_{13} \otimes\left|\Phi^{+}\right\rangle_{25} \otimes(\alpha|10\rangle+\beta|11\rangle-\gamma|00\rangle-\delta|01\rangle)_{46} \\
& +p_{1}|00\rangle_{\operatorname{ma}} \otimes\left|\Psi^{-}\right\rangle_{13} \otimes\left|\Phi^{-}\right\rangle_{25} \otimes(\alpha|10\rangle-\beta|11\rangle-\gamma|00\rangle+\delta|01\rangle)_{46} \\
& +p_{1}|00\rangle_{m n} \otimes\left|\Psi^{-}\right\rangle_{13} \otimes\left|\Psi^{+}\right\rangle_{25} \otimes(\alpha|11\rangle+\beta|10\rangle-\gamma|01\rangle-\delta|00\rangle)_{46} \\
& +p_{1}|00\rangle_{m a} \otimes\left|\Psi^{-}\right\rangle_{13} \otimes\left|\Psi^{-}\right\rangle_{25} \otimes\left(\alpha|11\rangle-\beta|10\rangle-\gamma|01\rangle+\delta|00\rangle_{46}\right. \\
& \left.+p_{2}|10\rangle_{m n} \otimes|00\rangle_{34} \otimes\left|\Phi^{+}\right\rangle_{25} \otimes(\alpha|00\rangle+\beta|01\rangle+\gamma|10\rangle+\delta \mid 11)\right\rangle_{16} \\
& +p_{2}|10\rangle_{m n} \otimes|00\rangle_{34} \otimes\left|\Phi^{-}\right\rangle_{25} \otimes(\alpha|00\rangle-\beta|01\rangle+\gamma|10\rangle-\delta|11\rangle)_{16} \\
& +p_{2}|10\rangle_{m n} \otimes|00\rangle_{34} \otimes\left|\Psi^{+}\right\rangle_{25} \otimes(\alpha|00\rangle+\beta|01\rangle+\gamma|10\rangle+\delta|11\rangle\rangle_{16} \\
& +p_{2}|10\rangle_{m n} \otimes|00\rangle_{34} \otimes\left|\Psi^{-}\right\rangle_{25} \otimes(\alpha|00\rangle-\beta|01\rangle+\gamma|10\rangle+\delta|11\rangle\rangle_{16} \\
& +p_{3}|01\rangle_{m n} \otimes|00\rangle_{56} \otimes\left|\Phi^{+}\right\rangle_{13} \otimes(\alpha|00\rangle+\beta|01\rangle+\gamma|10\rangle+\delta|11\rangle\rangle_{24} \\
& +p_{3}|01\rangle_{m m} \otimes|00\rangle_{56} \otimes\left|\Phi^{-}\right\rangle_{13} \otimes(\alpha|00\rangle-\beta|01\rangle+\gamma|10\rangle-\delta|11\rangle\rangle_{24} \\
& +p_{3}|01\rangle_{m n} \otimes|00\rangle_{56} \otimes\left|\Psi^{++}\right\rangle_{13} \otimes(\alpha|00\rangle+\beta|01\rangle+\gamma|10\rangle+\delta|11\rangle\rangle_{24} \\
& +p_{3}|01\rangle_{m n} \otimes|00\rangle_{56} \otimes|\Psi-\rangle_{13} \otimes(\alpha|00\rangle-\beta|01\rangle+\gamma|10\rangle+\delta|11\rangle\rangle_{24} \\
& +p_{4}|11\rangle_{m n} \otimes|00\rangle_{34} \otimes|00\rangle_{56} \otimes(\alpha|00\rangle+\beta|01\rangle+\gamma|10\rangle+\delta|11\rangle)_{12}
\end{aligned}
$$

Here $\left|\Phi^{ \pm}\right\rangle=\sqrt{2} / 2(|00\rangle \pm|11\rangle)$ and $\left|\Psi^{ \pm}\right\rangle=\sqrt{2} / 2(|01\rangle \pm|10\rangle)$. The probability factors $p_{k}(k=1,2,3,4)$ 
are expressed as:

$$
\begin{array}{cc}
p_{1}=b d / 2 & p_{2}=a d \sqrt{1-|b / a|^{2}} \\
p_{3}=c b \sqrt{1-|d / c|^{2}} & p_{4}=p_{2} \cdot p_{3}
\end{array}
$$

Step 3 The sender Alice measures the states of auxiliary particles $m$ and $n$, and the states of particles $(1,3)$ and $(2,5)$ using Bell measurements $\left[\left|\Phi^{ \pm}\right\rangle,\left|\Psi^{ \pm}\right\rangle\right]$. Then, the sender Alice will measure results through the classical channel to inform the receivers Bob and Charlie of the measurement results via classical channel.

\begin{tabular}{|c|c|c|c|c|c|}
\hline \multicolumn{3}{|c|}{ the measurement results } & \multirow{2}{*}{ Probabilities } & \multirow{2}{*}{$U_{e}$} & \multirow{2}{*}{$U_{C}$} \\
\hline$m$ and $n$ & 1 and 3 & 2 and 5 & & & \\
\hline \multirow{4}{*}{$|00\rangle_{m n}$} & \multirow{4}{*}{$\left|\Phi^{+}\right\rangle_{13}$} & $\left|\Phi^{+}\right\rangle_{25}$ & $|b d|^{2} / 4$ & $I$ & $I$ \\
\hline & & $\left|\Phi^{-}\right\rangle_{25}$ & $|b d|^{2} / 4$ & $I$ & $\sigma_{x}$ \\
\hline & & $\left|\Psi^{+}\right\rangle_{25}$ & $|b d|^{2} / 4$ & $I$ & $\sigma_{z}$ \\
\hline & & $\left|\Psi^{-}\right\rangle_{25}$ & $|b d|^{2} / 4$ & $I$ & $i \sigma_{y}$ \\
\hline \multirow{4}{*}{$|00\rangle_{m n}$} & \multirow{4}{*}{$\left|\Phi^{-}\right\rangle_{13}$} & $\left|\Phi^{+}\right\rangle_{25}$ & $|b d|^{2} / 4$ & $\sigma_{*}$ & $I$ \\
\hline & & $\left|\Phi^{-}\right\rangle_{25}$ & $|b d|^{2} / 4$ & $\sigma_{*}$ & $\sigma_{x}$ \\
\hline & & $\left|\Psi^{+}\right\rangle_{25}$ & $|b d|^{2} / 4$ & $\sigma_{x}$ & $\sigma_{x}$ \\
\hline & & $\left|\Psi^{-}\right\rangle_{25}$ & $|b d|^{2 / 4}$ & $\sigma_{x}$ & $i \sigma_{y}$ \\
\hline \multirow{4}{*}{$|00\rangle_{m n}$} & \multirow{4}{*}{$\left|\Psi^{+}\right\rangle_{13}$} & $\left|\Phi^{+}\right\rangle_{25}$ & $|b d|^{2} / 4$ & $\sigma_{z}$ & $I$ \\
\hline & & $\left|\Phi^{-}\right\rangle_{25}$ & $|b d|^{2} / 4$ & $\sigma_{z}$ & $\sigma_{n}$ \\
\hline & & $\left|\Psi^{+}\right\rangle_{25}$ & $|b d|^{2} / 4$ & $\sigma_{\bar{z}}$ & $\sigma_{z}$ \\
\hline & & $\left|\Psi^{-}\right\rangle_{25}$ & $|b d|^{2 / 4}$ & $\sigma_{z}$ & $i \sigma_{y}$ \\
\hline \multirow{4}{*}{$|00\rangle_{m n}$} & \multirow{4}{*}{$\left|\Psi^{-}\right\rangle_{13}$} & $\left|\Phi^{+}\right\rangle_{25}$ & $|b d|^{2} / 4$ & $i \sigma_{y}$ & $I$ \\
\hline & & $\left|\Phi^{-}\right\rangle_{25}$ & $|b d|^{2} / 4$ & $i \sigma_{y}$ & $\sigma_{x}$ \\
\hline & & $\left|\Psi^{+}\right\rangle_{25}$ & $|b d|^{2} / 4$ & $i \sigma_{y}$ & $\sigma_{z}$ \\
\hline & & $\left|\Psi^{-}\right\rangle_{25}$ & $|b d|^{2} / 4$ & $i \sigma_{y}$ & $i \sigma_{y}$ \\
\hline$n$ or $|1\rangle$ & & & $1-4|b d|^{2}$ & & \\
\hline
\end{tabular}

Table 1 the measurement results and the corresponding forms of unitary transformation

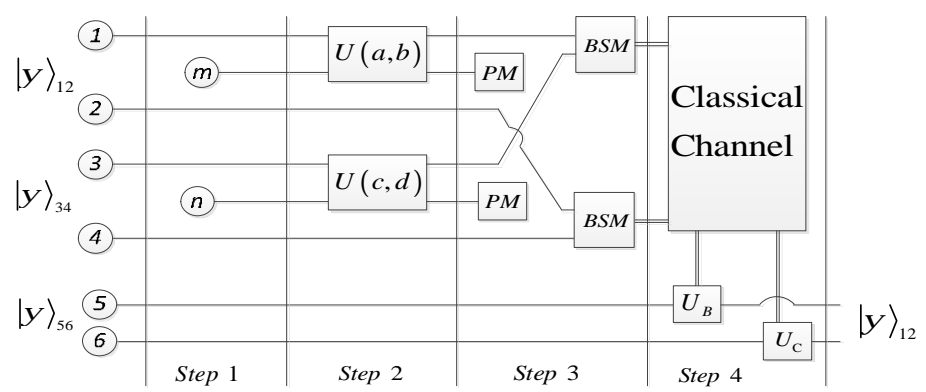

Fig. 1 This implementation process of this protocol

Step 4 For the teleportation of the state in Eq. (1), based on the results of the sender Alice, the 
receivers Bob and Charlie performs the corresponding unitary transformation $U_{S}$ and $U_{C}$ on their particles 4 and 6, respectively. Table 1 shows the measurement results and the corresponding specific forms of unitary transformation. Thus the teleportation of two-particles is realized. This implementation process of this protocol is shown in Fig. 1.

\section{The success probability and classic information}

In the research field of quantum teleportation, the success probability and classic information are important parameters. In this section, these two parameters would been analyzed. By Eq. (6), it can be seen that the auxiliary particle $m$ is $|1\rangle_{3 n}$, particles of $(3,4)$ would collapse to the state $|00\rangle_{34}$, thus the entanglement would be disappeare. Similarly, when the auxiliary particle $n$ is $|1\rangle_{n}$, particles of $(5,6)$ would be $|00\rangle_{56}$, and the teleportation could not be realized. So that, if and only if the auxiliary particle $m$ and $n$ are $|00\rangle_{m n}$, one can achieve the teleportation of two-particles. For auxiliary particles $m$ and $n$ under the state $|00\rangle_{m n}$, the probability of each component is equal to $\left(p_{1}\right)^{2}=|b d|^{2} / 4$, and the components are 16 , thus the total successful probability is equal to:

$$
P_{\text {total }}=16 \cdot|b d|^{2} / 4=4|b d|^{2}
$$

When $|a|=|b|=|c|=|d|=\sqrt{2} / 2$, the overall probability is equal to one, thus one can achieve the teleportation of two-particles with probability one. On the other hand, in the scheme of quantum teleportation, the classical information cost can be expressed as follows:

$$
c_{\text {total }}=16 \cdot\left[-|b d|^{2} / 4 \cdot \log _{2}\left[|b d|^{2} / 4\right)\right]=-4|b d|^{2} \cdot \log _{2}\left(\frac{|b a|^{2}}{4}\right) \quad b i t(s)
$$

When the quantum entanglement channel is formed by the maximum entangled state, namely, $|a|=|b|=|c|=|d|=\sqrt{2} / 2$, one need 4 bits classical information to achieve the teleportation of an arbitrary two-particle state. This result is consistent with the results of References [4, 9].

\section{Conclusion}

This paper presents a novel proposal to realize the probabilistic teleportation of two-particle states. Quantum channel is composed of two partially entangled states. By using this scheme, one can perform the teleportation under the condition that only the sender has the information of non-maximally entangled states. After the successful completion of the teleportation, the two receivers jointly own the original two-particle quantum state. At the same time, the concrete implementation processes of this proposal are given in detail, meanwhile the classical communication cost and the successful probability of our scheme are calculated. The above work for quantum teleporation research has reference value.

\section{References:}

[1] Bennett C H, Brassard G, Crepeau C, et al. Teleporting an unknown quantum state via dual classical and Einstein-Podolsky-Rosen channels [J]. Physical ReviewLetters.1993, 70: 1895-1899.

[2] Bouwmeester D, Pan JW, Mattle K, et al. Experimental quantum teleportation [J].Nature. 1997, 390: 575.

[3] Jiang M, Huang X, Zhou L L. An efficient scheme for multi-party quantum statesharing via non-maximally entangled states [J]. Chinese Science Bulletin. 2012,57 (10): 1089-1094.

[4] Wei J H, Dai H Y, Zhang M. A new scheme for probabilistic teleportation and itspotential applications [J].Communications in Theoretical Physics. 2013, 60 (6):651-657.

[5] Chiribella G, Giovannetti V, Maccone L, et al. Teleportation can only transfer speakable quantum information [J]. Physical Review A. 2010, 86: 010304(R).

[6] Townsend P D, Rarity J G, Tapster P R. Single photon interference in $10 \mathrm{~km}$ longoptical fibre interferometer [J]. Electronics Letters. 1993, 29 (7): 634-635.

[7]Li W L, Li C F, Guo G C. Probabilistic teleportation and entanglement matching [J]. Physical Review A. 2000, 61: 034301.

[8] Dai H Y, Chen P X, Li C Z. Probabilistic teleportation of an arbitrary two-particlestate by two partial three-particle entangledWstates [J]. Journal of Optics B. 2004,20 (8): 1196-1198. 
[9] Dai H Y, Chen P X, Li C Z. Probabilistic teleportation of an arbitrary two-particlestate by a partially entangled three-particle GHZ state andWstate [J]. Optics Communications. 2004, 231 (1-6): 281-287.

[10] Al-amri M, Evers J, Ikram M, et al. Quantum teleportation of high-dimensionalatomic ensemble states [J]. Journal of Physics B. 2012, 45 (9): 95502. 